\title{
STUDY AND RESEARCH ON IOT AND BIG DATA ANALYSIS FOR SMART CITY DEVELOPMENT
}

\author{
HAIXIA YU* ION COSMIN MIHAI † AND ANAND SRIVASTAVA
}

\begin{abstract}
With the development of smart meters, like Internet of Things (IoT), various kinds of electronic devices are equipped with each smart city. The several aspects of smart cities are accessible and these technologies enable us to be smarter. The utilization of the smart systems is very quick and valuable source to fulfill the requirement of city development. There are interconnection between various IoT devices and huge amount of data is generated when they communicate each other over the internet. It is very challenging task to effectively integrate the IoT services and processing big data. Therefore, a system for smart city development is proposed in this paper which is based on the IoT utilizing the analytics of big data. A complete system is proposed which includes various types of IoT-based smart systems like smart home, vehicular networking, and smart parking etc., for data generation. The Hadoop ecosystem is utilized for the implementation of the proposed system. The evaluation of the system is done in terms of throughput and processing time. The proposed technique is $20 \%$ to $65 \%$ better than the existing techniques in terms of time required for processing. In terms of obtained throughput, the proposed technique outperforms the existing technique by $20 \%$ to $60 \%$.
\end{abstract}

Key words: Internet of Things; Smart city development; Processing time, Throughput; Smart systems

AMS subject classifications. $68 \mathrm{M} 14$

1. Introduction. Nowadays, with the advancement in technologies, there are many requirements and needs that are required to be full-filled. The Information technology is required always when we look at peculations in each and every form. In many community services like local departments, law enforcement, public libraries and learning institutions, there is always ease of access [1, 2]. Alternatively, the mitigated accesses of all the devices are encapsulated by Internet of Things (IoT). In the same way, to improve the outputs at greater levels and in order to pace up the accessibility, devices are meant to be automated. The automatization of the devices can be done by utilizing a single device like wrist watch etc [3]. The sensors can be used to automate the daily life operations for saving the human efforts and also time collaboration is reduced. The new advantages are added by the large data sets to the table in which high speed and efficiency is required. In previous time, the information is gathered, analyzed and updated by the companies for easy retrieval for decision making in future but nowadays, for faster decisions, the companies can easily find ideas [4, 5]. The faster processing is the greatest advantage in the companies nowadays that is never existed before. It is very important to deal with the big data and the quick and accurate decision should be the main goal. The clustering and a better understanding of the information led by the industrial awareness which can be controlled and the deviation analysis are performed from the patterns that can influence the business process outcomes. For the economic growth, the speculation of the whole smart cities is done in the development of urban areas which are feasible to rectify the technological dependent issues [6-8]. For digitized information exchange, the big data and Internet of Things (IoT) are utilized by the smart cities for the city services improvement w.r.t performance and wellbeing of citizens. The management of the urban flow is improved while developing the smart city applications. With time, the population in cities is expected to get doubled by 2060 . The enormous pressure is increased by it. The integration of IoT and big data has the significant impression and implanted in the smart devices and directly or indirectly make available to billion people $[9,10]$. The various inferences are deducted by applying the big data analytics. The quality of people's lives standards are raised by the IoT

*Hefei Technology College, Hefei 230012, China (haixiayu1212@outlook.com).

$\dagger$ The Faculty of Police, "AI.I.Cuza" Police Academy, Romania (cosmin.mihai@yahoo.com ).

†ABES Engineering College Ghaziabaad, India (anand8355@gmail.com). 
and big data.

The enormous volume of structured/unstructured data is described by the Big Data which is managed by utilizing the traditional databases. The exponential growth and availability is described by the big data and utilize the structured and unstructured information [11]. From smart cities applications, big data system will stock in an efficient way and the enhanced information is provided regarding the smart city services. The decision-makers are helped by this enhanced information in various smart city services. The volume, variety, velocity, variability and value are the different features of big data. The amount of data is referred by the volume feature. The structured and unstructured data is included in the variety feature of big data which is diverse in nature and from multiple sources. The velocity refers the speed which shows the flow of big data from diverse sources. In the structure and meaning of data, there is a constant change which is showed by the variability. The value feature of the big data shows the big data advantages which is provided to the business [12]. Hence, huge potential is depicted by the different features for advancements bounded by the available technologies to achieve the goals in smart cities. The correct tools and methods are required by the big data that give efficient results.

1.1. Challenges of big data and IOT in smart cities. As the smart cities are developing, there are many challenges that are faced while utilizing the big data and IoT during smart city implementation. The multiple sources and their formats are the biggest challenge and utilization of the traditional software for data processing is very difficult. The data integration is the challenge from different environments and intelligent devices. When information is passed, the scenario becomes more challenging and the privacy of the people's lives is prevented by the smart city solution [13]. The various organizations collect the data and data maintenance is very challenging. Without tampering the data quality, data is required to store in specific format with the incorporation of big data. In the big data environment the security of the data is also challenging as the data size is very high. A secured smart city is designed and the data breaching required be avoiding and properly encrypting [14]. With time and advancement in the technology, the smart city is becoming smarter than the past. Different types of electronic equipment are consisted in the smart cities applied by the applications, such as monitoring system's cameras and transportation system's sensors and so on. The individual mobile equipment is spreading with time. The different aspects of smart cities are smart buildings, smart technology, and smart infrastructure. The features of a smart city are shown in Figure 1.1.

According to geographical position, device aggregation is done in an IoT environment and it is assessed by applying analyzing systems. The utilization of the specific data by sensor services with the projects regarding the monitoring of each cyclist [15]. An IoT substructure is utilized by the lot of service domain applications for simplification of the air operations and noise pollution control as well as surveillance systems.

The aim of this paper is to propose a smart city development which is based on the IoT utilizing the analytics of big data. A complete system is proposed which includes various types of IoT-based smart systems like smart home, vehicular networking, and smart parking etc., for data generation. The rest of the paper is organized as follows. Section 2 provides an overview of the exhaustive literature survey followed by a methodology adopted in section 3. A detailed discussion of obtained results is in section 4. Finally, concluding remarks and future directions are provided in Section 5.

2. Literature Review. Nowadays, there are rapid evolvement of the Internet of Things (IoT) technologies and big data [16]. Hence major role is played for development of smart cities. A perfect blend is formed by the IoT and big data in fetching an interesting and novel challenge for smart cities. The business and technology related issues are focused by these new challenges that help smart cities for principles and vision requirement formulation. Authors in this paper analyze the big data role and the IoT technologies w.r.t smart cities. The benefits of big data and IoT in smart cities are also discussed by the author. In the new era, enhancement of the technological aspects is done with the rusty flaws mitigation and it encapsulates in a wide range of methodologies [17]. The human efforts are focused in daily based tasks done in the city and an enthusiastic solution is provided for the maximum level of ease incorporation. The automation of such tasks is involved in it to save time and ensure the productivity. The idea of automation is incorporated in city and the mechanism is controlled through the automation system like sensors etc. The bulk of data is there in form of database, device operational attributes and user responses and it is called IoT environment. The services provision and infrastructure is demanded by the population rapid growth in urban areas [18]. The IoT devices are utilized by 


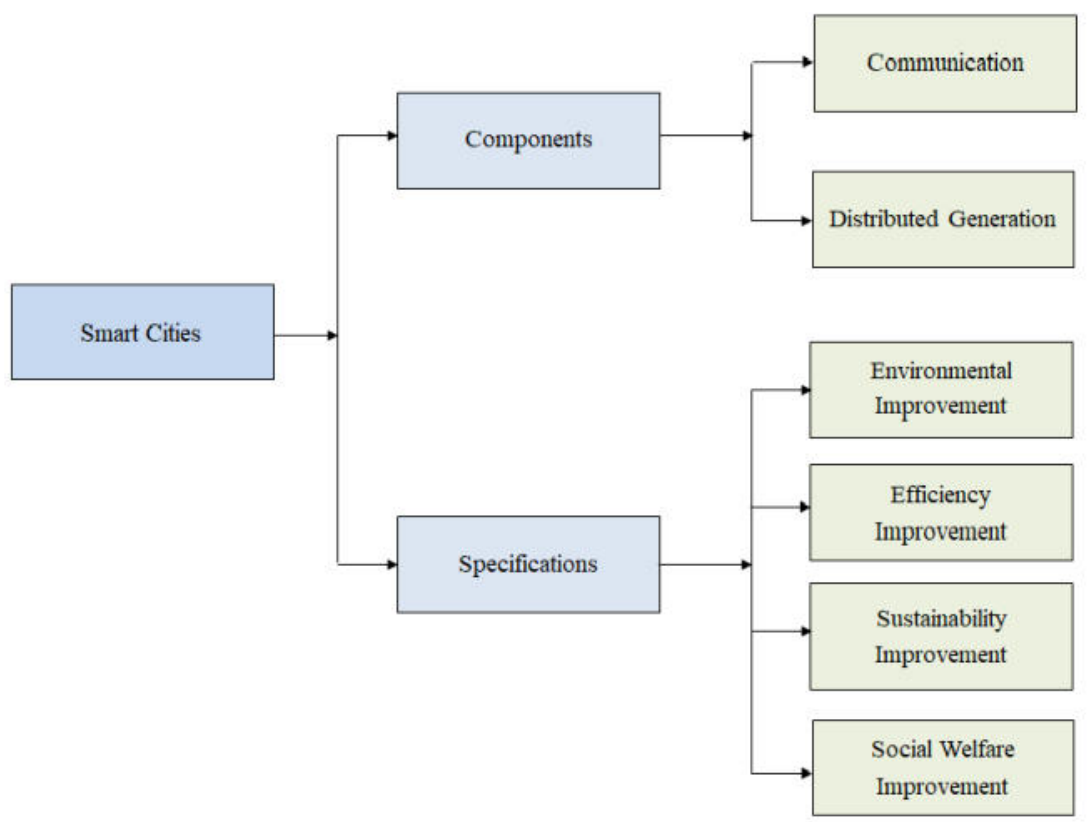

FIG. 1.1. Key aspects of smart city

it like sensors, actuators and smart systems. There is interconnection between thousands of IoT devices and they communicate with each other for smart systems establishment and the huge amount of data is known as big data. The integration of IoT services and Big Data process in an effective way is the challenging task. An IoT-based system is presented in this paper for planning super city by utilizing the analytics of big data. A complete system is proposed by the authors and includes smart systems like smart home, weather and water system. The efficiency of the presented technique is shown by the throughput and processing time. The smart city concept is adopted by many governments in their cities and big data applications are implemented that supports the components of smart city for the improvement of living standards [19]. The performance of health, transportation, energy, education, and water services are improved by the smart cities by utilizing the multiple techniques. The cost reduction and resource consumption is involved in it for more efficiently engaging with citizens. For the enhancement of smart city, big data analytics is the recent technology which is having high potential. Nowadays, almost everything is digital and collection of large data in the huge amounts of data accumulation can be utilized in various beneficial application domains. In many business and service domains, big data utilization and analysis is the key factor for success. The more facilities and resources are demanded by the large population density in urban areas. The Internet of Things (IoT) devices and the smart systems are utilized as the quick and valuable source to meet the requirements for the development of city [20]. Huge amount of data is generated by interconnecting the IoT devices and the Big Data. The IoT system using big data analytics is presented by the author for the development of smart cities. Various researchers utilize the existing datasets by including smart homes, pollution and vehicle for analysis and testing. The real-time system efficiency is tested by utilizing all the datasets and finally the system is evaluated in terms of throughput and processing time. The various kinds of electronic devices are equipped with the smart city with the smart meters expansion like Internet of Things (IoT) [21]. Therefore, technologies enable us to make smart cities more accessible and applicable. An inclusive review on the concept of the smart city is provided in this paper with their benefits. All across the world, implementation's key barriers are expressed thoroughly. The solutions of the smart cities are implemented monolithically from the sensors data through to the provided services [22]. The different developers faced the same challenges in a new city. The methodologies are presented in this paper for the minimization of the efforts for the new smart city implementation and the component sharing 
are maximizing. The atomic service examples are detailed in this paper with some data predictor components. Furthermore, real-world atomic services are described by the usage in the scenarios of Santander. A side market is also generated by the atomic services that allow the expertise to be re-used by different stakeholders. The ability to management and remotely monitor is provided by the IoT from huge streams of real-time data [23]. A scalable IoT video data is offered by the model for smart cities for scalability exploitation to execute analysis on large datasets. The data analytics programming are provided by the model in which scalable analytics services are designed by the authors. The video analysis process is automated by the system and the manual intervention is reduced. The development of the design model is extended for IoT devices, message routing and data analytics. The Internet of things (IoT) in the smart city is examined, managed and controlled by the proper utilization of resources in smart cities [24]. The applications of IoT are used in the smart cities without human involvement [24]. There are interconnection between the IoT devices which communicate for different tasks. The huge volume of data is produced by billions of devices connected on IoT that is bound to cloud for processing and storage. The diverse research directions are produced by the IoT evolution for the smart city. A SOA (Service Oriented Architecture)-based platform is presented by the authors in this paper that enables the big datasets stemming social networking (SN) sites and IoT [25]. The application of data analytics are studied in this paper like sentiment analysis saved into an SQL database and algorithms are further investigated for minimization of dataset processing delays. A new era in urban research, planning and policy are defined by the big data analysis. The pattern detection and real-time data mining can now be carried out at a large scale [26]. The limitations of big data for urban policy and planning are discussed in this paper. A theoretical perspective is developed on urban analytics as a practice which is a part of a smart urbanism.

3. Research Methodology. The IoT-based Super City planning system is established for data generation and analysis. For safety, the numerous sensors deployment and other fixed devices are presented. For Super City planning, existing smart systems data in the city is utilized. The abundant amount of big data is generated by the systems and the sensors at very high speed. The data is processed in an efficient way by utilizing the Hadoop ecosystem. The proposed architecture is demonstrated in this section containing the sensors deployment details and how the data is generated by the system. The integration of the IoT-based smart systems is done with the city data generation for the super city planning and generation as shown in Fig 3.1. For future Super City planning, analysis of smart systems' large data is done which is stored in the large storage. The electricity consumption of the previous years is analyzed by the government authorities which gives the electricity demand prediction by the generated data by the homes. To produce more energy, the future needs are accomplished by taking the action by the municipalities. For power bill management, the analysis of the power usuage patterns with different time periods is done at all houses [27-30]. For various time-periods, various energy plans can also be set and design the future plan from data analysis of smart parking and vehicular traffic.

Furthermore, air-pollution causes are identified and environmental data is analyzed which is utilized for find the places with toxic gases. The pollution is reduced by the plans made by authorities e.g., transferring factories, diverting traffic, etc. similarly, by weather and water resources datasets analysis. The better strategies are made for electricity saving during the hot seasons on the basis of electricity expenditure and temperature datasets [31]. The unsafe places, number of crimes events, number of victims, etc. are investigated by utilizing the historical surveillance data. A model is also designed by the researchers for the implementation of the system that guide the authorities for the urban planning performance by the proposed system implementation. The Fig 3.2 presented this model; the large data is generated by every smart system in the beginning by environmental sensing. By utilizing the Zig-Bee communication, there is a connection of the relay node with every smart system. The data is then transmitted by the relay node to the analytical system which is passing through the internet $[32,33]$. In the raw data, the majority information is the metadata from the sensors. Therefore, discarded all the irrelevant data and the sensor data has the message information and the identifier which is utilized for data classification. There is transformation of the classified data to the Hadoop readable file format like sequence file. The large amount of data is handled by it and an effective system is needed by the authors which process the huge amount of data speedily [34]. Hadoop ecosystem is deployed by the authors to fulfill the basic requirements and it is also equipped with master node and other nodes working under master node.

The large datasets are stored in the Hadoop ecosystem by utilizing the Hadoop Distributed File System 


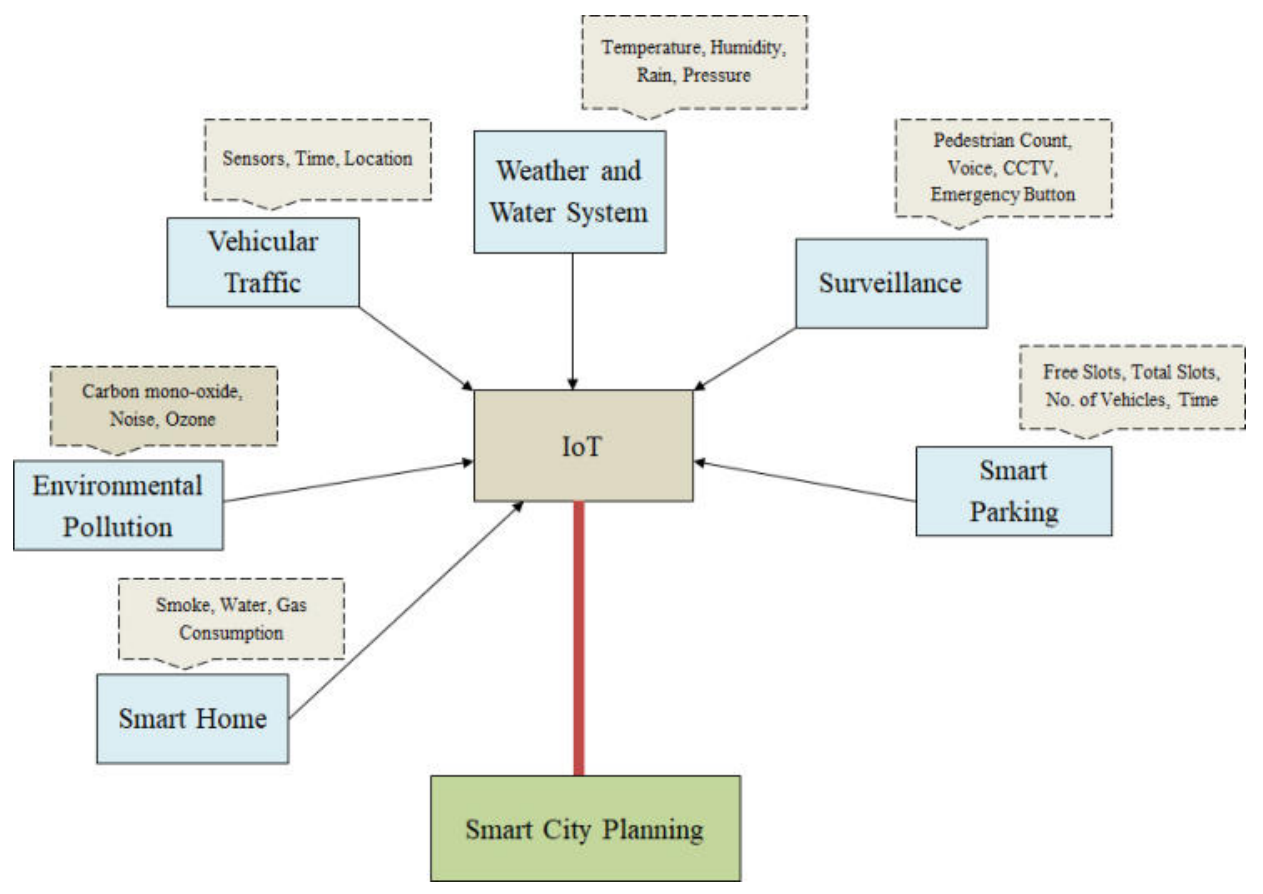

FIG. 3.1. Integration of the IoT-based smart systems with the city data generation

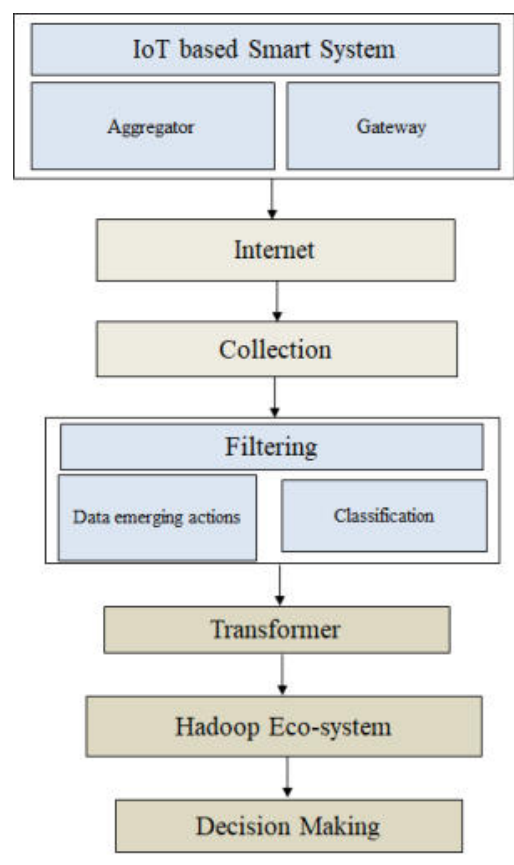

FIG. 3.2. IoT based smart city system

(HDFS) which divide them into equal sized chunks. These chunks are also stored by it in different nodes by duplicating every chunk on more than one data nodes for more safety and reliability achievement. The processing of these chunks is in parallel for performance analysis. All the generated chunks are then aggregated by utilizing the Hadoop eco-system reduce function. The decision making is then finally performed based on 
TABLE 4.1

Processing time of various increasing size datasets

\begin{tabular}{||l|l||}
\hline Dataset Size & Processing Time \\
\hline \hline 70 & 20000 \\
\hline 150 & 25000 \\
\hline 300 & 30000 \\
\hline 450 & 40000 \\
\hline 1220 & 55000 \\
\hline 1840 & 130000 \\
\hline 3270 & 190000 \\
\hline 5340 & 240000 \\
\hline
\end{tabular}

the Hadoop ecosystem's results. The pattern recognition, soft computing models are utilized for the decision making approach.

4. Results and Discussion. For data generation, implementation of smart systems is very difficult at this time. So, from various reliable resources, already generated datasets are taken through the smart systems. There are different datasets which includes the flood dataset details the flood occurrence, the temperature and water usage of house collected by the smart home sensors, vehicle's information such as speed etc. The incessant measurements of heat, moisture, humidity, rain, etc. are included in the weather datasets.

By the parallel processing, huge amount of data is accomplished through Hadoop and analysis of such large data is really a challenging task. The government authorities are not only beneficial by the proper super city planning but with high security and healthy environment, it also benefits the public. For urban development various kinds of data is analyzed by authors and guidelines are given to the consultants that how the IoT technologies and the Big Data are utilized produced from IoT. By utilizing the presented system and the data analysis, the super city is achieved by the authorities for the citizens of the next generation. Mainly, vehicular traffic, smart home, flood, and pollution are focused for analysis. In Super City planning, analysis of smart home data played very important role including energy and water management, house building structure planning etc. The authors are presenting the water management analysis and individual house usage based planning. The water meter readings of 60324 houses and the reading period of house are consisted in the database. By analyzing the normal utilization of water at house, future need of water can be predicted. More than 7000-8000 cubic meters are consumed by more than 5000 houses as obtained by the analysis. The area wise usage of water is also analyzed from where flow of water is controlled by the authorities depending on the area requirement.

4.1. System Evaluation. The processing of big datasets is the main focus for the development of the system. The system effectiveness is focused by the authors while performing the implementation and evaluation of the system. The various datasets are taken for the system efficiency evaluation. The various heterogeneous datasets are merged into the single dataset to make the complex dataset. The system throughput and the processing time are the two main parameters to evaluate the system and measuring the system efficiency. The processing time of various datasets is tabulated in Table 4.1 which is also presented graphically in Figure 4.1. The Table 4.2 depicts the throughput results and graphical representation is shown in Figure 4.2. The time of processing is based on the datasets. Throughput of the system is inversely related to the processed data volume. The parallel processing Hadoop ecosystem environment results the inversely proportional throughput.

On the nation development, there is key influence of the city planning and decision control is increased. The IoT is utilized for planning the super city and the proposed implementation model is analyzed by utilizing the Hadoop. The functionality of data aggregation, processing and management is devised by the four tired architecture. The different smart systems generate the various datasets that are utilized for the analysis of the urban data. Data investigation and evaluation is also done. On the life of the citizens of coming generations, there is great impact of the presented system. Then the complex system is presented for planning of the super city by utilizing the IoT. The big data generated by the smart system is processed and the presented implementation model analyzed it by utilizing the Hadoop. The functionality of data aggregation, 


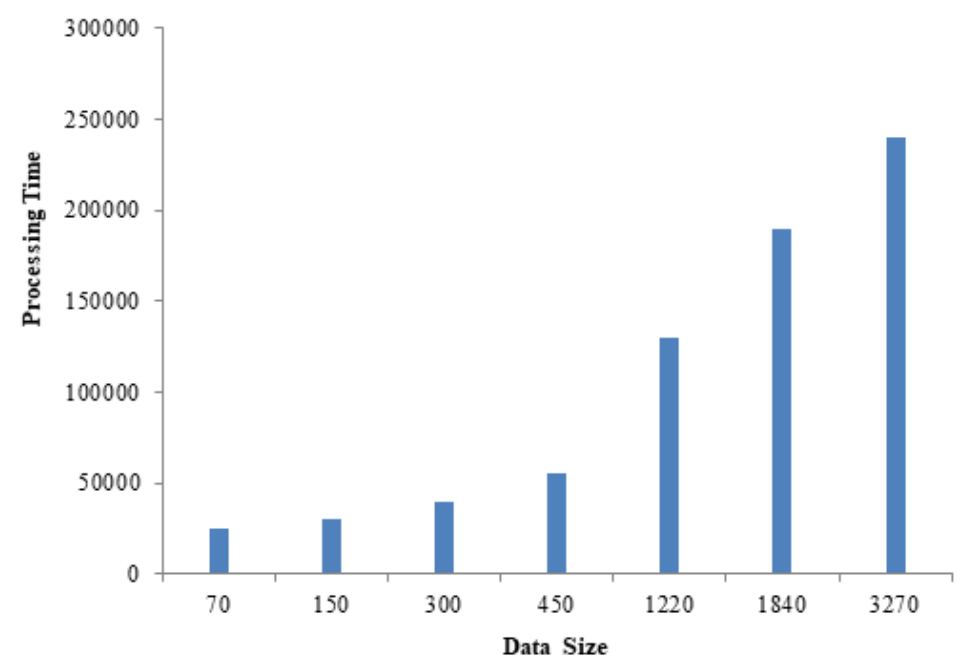

FIG. 4.1. System processing time (sec) on increasing data size

TABLE 4.2

System throughput for increasing size datasets

\begin{tabular}{||l|l||}
\hline Dataset & Throughput (MBps) \\
\hline \hline 100 & 5 \\
\hline 200 & 7 \\
\hline 300 & 8 \\
\hline 600 & 9 \\
\hline 1200 & 10 \\
\hline 1800 & 15 \\
\hline 3200 & 19 \\
\hline 5300 & 22 \\
\hline
\end{tabular}

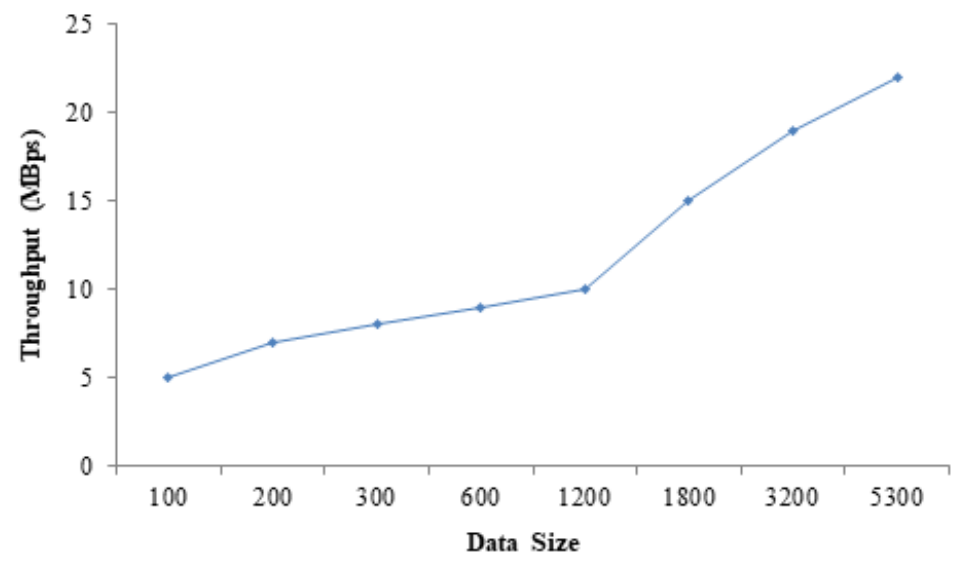

FIG. 4.2. System throughput on increasing data size

data processing and management is devised by the four tire architecture. On the coming generation's life, there is a major impact of the presented system and many facilities and needs are provided by the system. Based 


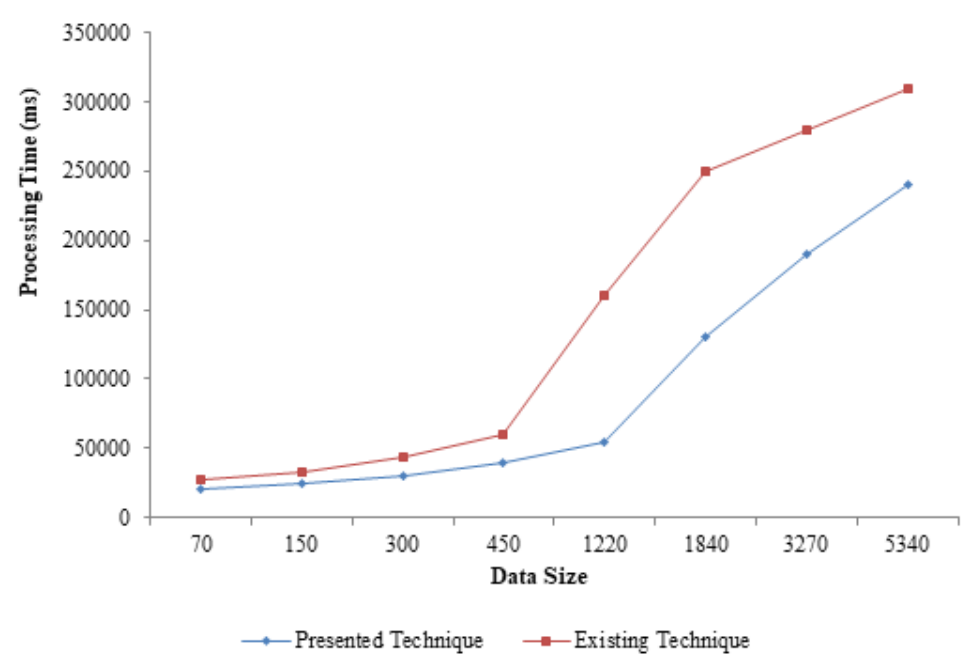

FIG. 4.3. Proposed and existing system's processing time on increasing data size

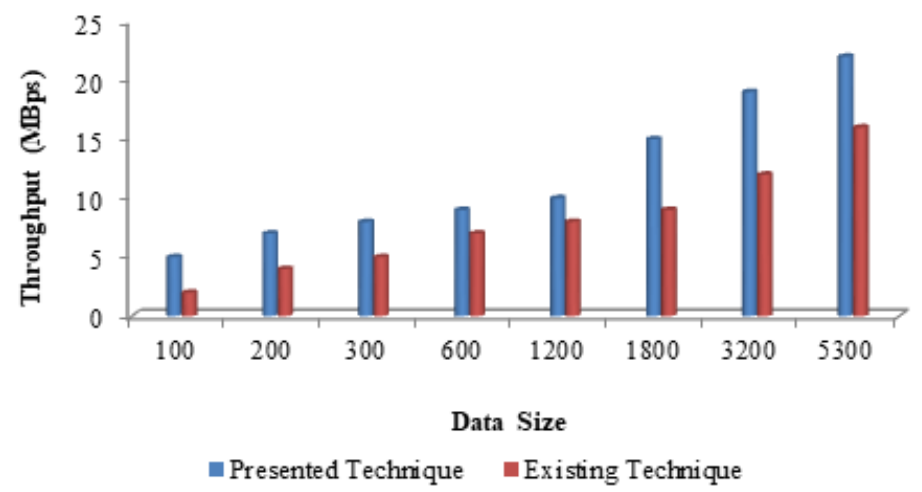

FIG. 4.4. Proposed and existing system's throughput on increasing data size

on the performance efficiency, the evaluation of the system is done and the system throughput and processing time are also focused. The performance of the system is extraordinary when large datasets are processed. The throughput of the system is increases with the increase of the data size.

4.2. Comparison of the presented Technique with the existing Technique. The performance of the proposed technique is also compared with the existing technique [18] for evaluation purpose. The comparison is done in terms of processing time taken by the system and the overall throughput obtained for different size of the data. The values of the processing time by the existing technique and the presented technique are presented in Figure 4.3 for better analysis and visualization.

It is clear from the Figure 4.3 that the processing time consumed by the proposed technique is less as compared to the existing technique. The presented technique is performed well while consuming less processing time as compared to the existing technique. The comparison is also made in terms of overall throughput obtained with the existing technique. The obtained throughput values obtained by the presented technique and the existing technique are presented graphically in Figure 4.4.

It is obtained from the Figure 4.4 that the proposed technique gives high throughput in comparison with the existing technique. The proposed technique performs well in terms of throughput while consuming the less 


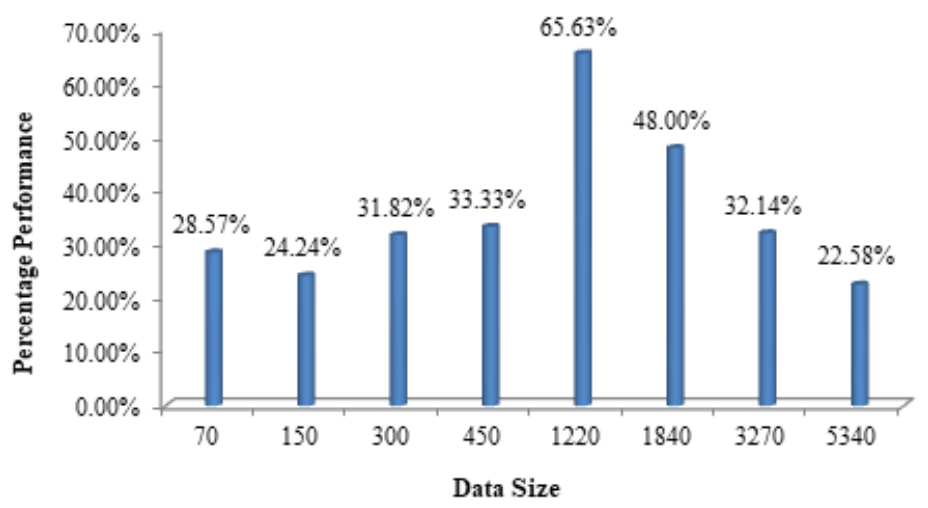

FIG. 4.5. The percentage improvement of the proposed technique in terms of system's processing time

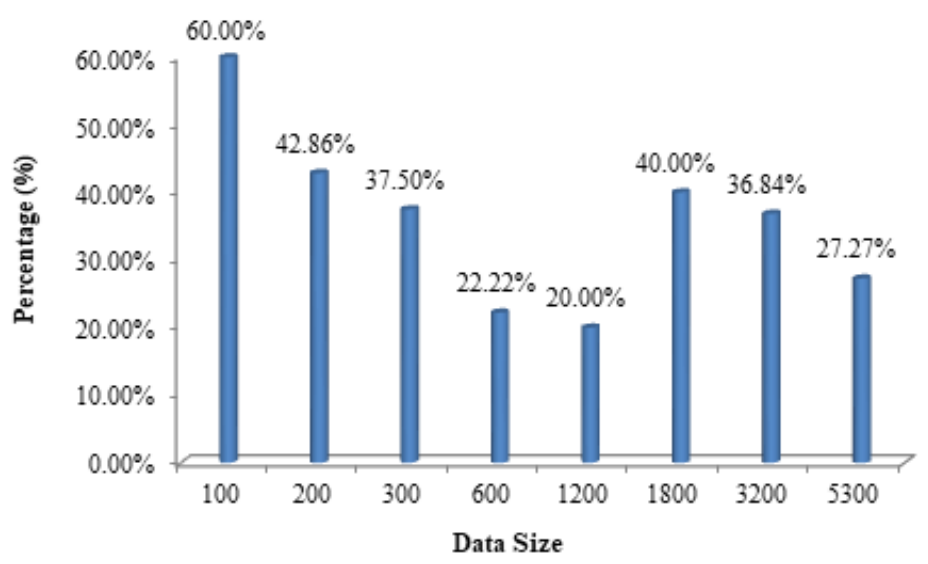

FIG. 4.6. The percentage improvement of the proposed technique in terms of system's throughput

processing time. The performance of the proposed technique is also evaluated by calculating the percentage of the proposed technique over the existing technique in terms of processing time and the obtained throughput. The graphical representation of percentage improvement of the proposed technique over the existing technique in terms of processing time is shown in Figure 4.5. The proposed technique is $20 \%$ to $65 \%$ better than the existing techniques in terms of time required for processing.

The percentage improvement of the proposed technique is also seen in terms of throughput over the existing technique. The graphical representation of the percentage improvement is shown in Figure 4.6.

It is clear from the Figure 4.6 that the high percentage performance is obtained by the proposed technique over the existing technique. The proposed technique is $20 \%$ to $60 \%$ better than the existing technique in terms of obtained throughput.

5. Conclusion and Future Work. The Information technology is required always when we look at peculations in each and every form. In many community services like local departments, law enforcement, public libraries and learning institutions, there is always ease of access. To facilitate the government as well citizens, an IoT-based system is presented in this paper. The government authorities are not only beneficial by the proper super city planning but with high security and healthy environment, it also benefits the public. Large amount of data is processed which is coming with high speed by the Hadoop ecosystem at the top of the layer. The system's efficiency is evaluated by utilizing the datasets of the existing smart systems. The 
performance of the proposed technique is also compared with the existing technique for evaluation purpose. The comparison is done in terms of processing time taken by the system and the overall throughput obtained for different size of the data. The presented technique is performed well while consuming less processing time as compared to the existing technique. The proposed technique is $20 \%$ to $65 \%$ better than the existing techniques in terms of time required for processing. In terms of obtained throughput, the proposed technique outperforms the existing technique by $20 \%$ to $60 \%$. The deployment of the system by utilizing practical smart systems will be in future directions. To achieve Super City, the smart systems can be integrated with the municipality's system in the future. The system security is also important as the high criticality of the system.

Acknowledgment. Key project of natural science research in Colleges and universities of Anhui Provincial Department of education in 2020 "research and design of online learning early warning system based on education big data" (No.: KJ2020A0990).

\section{REFERENCES}

[1] Mehmood, Y., Ahmad, F., Yaqoob, I., Adnane, A., Imran, M., and Guizani, S., Internet-of-things-based smart cities: Recent advances and challenges, IEEE Communications Magazine, 55(9), 16-24, 2017.

[2] Hashem, I. A. T., Chang, V., Anuar, N. B., Adewole, K., Yaqoob, I., Gani, A., ... and Chiroma, H., The role of big data in smart city, International Journal of Information Management, 36(5), 748-758, 2016.

[3] Atitallah, S. B., Driss, M., Boulila, W., and Ghézala, H. B., Leveraging Deep Learning and Iot big data analytics to support the smart cities development: Review and future directions, Computer Science Review, 38, 100303, 2020.

[4] Sun, Y., Song, H., JARA, A. J., AND Bie, R., Internet of things and big data analytics for smart and connected communities, IEEE access, 4, 766-773, 2016.

[5] Ang, L. M., Seng, K. P., Zungeru, A. M., and Ijemaru, G. K. , Big sensor data systems for smart cities, IEEE Internet of Things Journal, 4(5), 1259-1271, 2017.

[6] Plageras, A. P., Psannis, K. E., Stergiou, C., Wang, H., and Gupta, B. B. , Efficient Iot-based sensor BIG Data collection-processing and analysis in smart buildings, Future Generation Computer Systems, 82, 349-357, 2018.

[7] QIan, Y., Wu, D., BaO, W., And Lorenz, P. , The internet of things for smart cities: Technologies and applications, IEEE Network, 33(2), 4-5, 2019.

[8] Brohi, S. N., Bamiah, M. E. R. V. A. T., and Brohi, M. N., Big data in Smart Cities: a systematic mapping review, Journal of Engineering Science and Technology, 13(7), 2246-2270, 2018.

[9] Rizwan, P., Suresh, K., And Babu, M. R., Real-time smart traffic management system for smart cities by using Internet of Things and big data, In 2016 international conference on emerging technological trends (ICETT) (pp. 1-7). IEEE, 2016.

[10] Ge, M., Bangui, H., And Buhnova, B., Big data for internet of things: a survey, Future generation computer systems, 87, 601-614, 2018.

[11] Nosratabadi, S., Mosavi, A., Keivani, R., Ardabili, S., and Aram, F. , State of the art survey of deep learning and machine learning models for smart cities and urban sustainability, In International Conference on Global Research and Education (pp. 228-238). Springer, Cham, 2019.

[12] Rizwan, P., Suresh, K., And Babu, M. R. , Real-time smart traffic management system for smart cities by using Internet of Things and big data, In 2016 international conference on emerging technological trends (ICETT) (pp. 1-7). IEEE, 2016.

[13] Al-Turjman, F., Zahmatkesh, H., and Shahroze, R. , An overview of security and privacy in smart cities' IoT communications, Transactions on Emerging Telecommunications Technologies, e3677, 2019.

[14] Lloret, J., Tomas, J., Canovas, A., And Parra, L. , An integrated IoT architecture for smart metering, IEEE Communications Magazine, 54(12), 50-57, 2016.

[15] Soomro, K., Bhutta, M. N. M., Khan, Z., and Tahir, M. A. , Smart city big data analytics: An advanced review, Wiley Interdisciplinary Reviews: Data Mining and Knowledge Discovery, 9(5), e1319, 2019.

[16] Nagpal, N., Analyzing role of big data and iot in smart cities, International Journal of Advanced engineering, Management and Science, 3(5), 239858, 2017.

[17] Ahmed, F. , Implementation of smart cities under IoT and big data analytics, IJCSNS, 17(10), 153, 2013.

[18] Rathore, M. M., Paul, A., Ahmad, A., And Jeon, G., IoT-based big data: from smart city towards next generation super city planning, International Journal on Semantic Web and Information Systems (IJSWIS), 13(1), 28-47, 2017.

[19] Al Nuaimi, E., Al Neyadi, H., Mohamed, N., And Al-Jaroodi, J. , Applications of big data to smart cities, Journal of Internet Services and Applications, 6(1), 1-15, 2015.

[20] Ganesh, E. N., Development of Smart City Using IOT and Big Data, Int. J. Comput. Tech, 4(1), 36-37, 2017.

[21] Talari, S., Shafie-Khah, M., Siano, P., Loia, V., Tommasetti, A., and Catalão, J. P., A review of smart cities based on the internet of things concept, Energies, 10(4), 421, 2017.

[22] Cirillo, F., Gómez, D., Diez, L., Maestro, I. E., Gilbert, T. B. J., And Akhavan, R., Smart city Iot services creation through large-scale collaboration, IEEE Internet of Things Journal, 7(6), 5267-5275, 2020.

[23] Doan, M. P., Huynh, H. H., And Huynh, H. X. , Design of IoT blockchain based smart agriculture for enlightening safety and securityA Scalable IoT Video Data Analytics for Smart Cities, EAI Endorsed Transactions on Context-aware Systems and Applications, 6(19), 2019. 
[24] Huang, C., And Nazir, S., Analyzing and Evaluating Smart Cities for IoT Based on Use Cases Using the Analytic Network Process, Mobile Information Systems, 2021.

[25] Psomakelis, E., Aisopos, F., Litke, A., Tserpes, K., Kardara, M., and Campo, P. M., Big Iot and social networking data for smart cities: Algorithmic improvements on Big Data Analysis in the context of RADICAL city applications, arXiv preprint arXiv:1607.00509, 2016.

[26] Kandt, J., And Batty, M. , Smart cities, big data and urban policy: Towards urban analytics for the long run, Cities, 109, 102992, 2021.

[27] Vilajosana, I., Llosa, J., Martinez, B., Domingo-Prieto, M., Angles, A., and Vilajosana, X. , Bootstrapping smart cities through a self-sustainable model based on big data flows, IEEE Communications magazine, 51(6), 128-134,2013.

[28] Ahmed, E., Yaqoob, I., Hashem, I. A. T., Khan, I., Ahmed, A. I. A., Imran, M., and Vasilakos, A. V. , The role of big data analytics in Internet of Things, Computer Networks, 129, 459-471,2017.

[29] ZekiĆ-SuŠAC, M., Mitrović, S., AND HAS, A., Machine learning based system for managing energy efficiency of public sector as an approach towards smart cities, International journal of information management, 58, 102074,2021.

[30] Ahmed, E., Yaqoob, I., Gani, A., Imran, M., and Guizani, M., Internet-of-things-based smart environments: state of the art, taxonomy, and open research challenges, IEEE Wireless Communications, 23(5), 10-16,2016.

[31] Romero, C. D. G., Barriga, J. K. D., and Molano, J. I. R., Big data meaning in the architecture of IoT for smart cities, In International Conference on Data Mining and Big Data (pp. 457-465). Springer, Cham,2016.

[32] Kang, G. K., GaO, J. Z., Chino, S., Lu, S., and Xie, G., Air quality prediction: Big data and machine learning approaches, International Journal of Environmental Science and Development, 9(1), 8-16,2018.

[33] He, Y., Yu, F. R., Zhao, N., Leung, V. C., And Yin, H., Software-defined networks with mobile edge computing and caching for smart cities: A big data deep reinforcement learning approach, IEEE Communications Magazine, 55(12), 31-37, 2017.

[34] Liu, Y., YAng, C., JiAng, L., Xie, S., And Zhang, Y., Intelligent edge computing for IoT-based energy management in smart cities, IEEE Network, 33(2), 111-117, 2019.

Edited by: Pradeep Kumar Singh

Received: May 30, 2021

Accepted: Sep 20, 2021 
